\title{
Gel Point Determination of TEOS-Based Polymeric Materials with Application on Conservation of Cultural Heritage Buildings
}

\author{
R. Angulo-Olais $\mathbb{D}^{1},{ }^{1}$ Juan F. Illescas, ${ }^{2}$ J. Aguilar-Pliego, ${ }^{3}$ \\ C. A. Vargas, ${ }^{3}$ and C. Haro-Pérez iD $^{3}$ \\ ${ }^{1}$ Programa de Doctorado en Ciencias e Ingeniería de Materiales, Universidad Autónoma Metropolitana-Azcapotzalco, \\ Av. San Pablo 180, 02200 CDMX, Mexico \\ ${ }^{2}$ Centro de Investigación en Corrosión, Universidad Autónoma de Campeche, Av. Agustín Melgar s/n, \\ 24039 San Francisco de Campeche, CAM, Mexico \\ ${ }^{3}$ Departamento de Ciencias Básicas, Universidad Autónoma Metropolitana-Azcapotzalco, Av. San Pablo 180, 02200 CDMX, Mexico
}

Correspondence should be addressed to C. Haro-Pérez; cehp@azc.uam.mx

Received 12 March 2018; Revised 7 May 2018; Accepted 5 June 2018; Published 5 July 2018

Academic Editor: Charles Rosenblatt

Copyright (c) 2018 R. Angulo-Olais et al. This is an open access article distributed under the Creative Commons Attribution License, which permits unrestricted use, distribution, and reproduction in any medium, provided the original work is properly cited.

Here we present measurements on the gel time of inorganic-organic materials used for stone preservation by means of rheology and dynamic light scattering. Our hybrid material is composed of tetraethyl orthosilicate (TEOS) and polydimethylsiloxane (PDMS) using a nonionic surfactant (n-octylamine) as a template. Moreover, zinc oxide $(\mathrm{ZnO})$ nanoparticles are dispersed in the medium with the aim of obtaining a nanocomposite with potential biocide properties. In our case, we use the $\mathrm{ZnO}$ particles as tracers to infer from their scattered intensity mechanical information of the suspending medium. We have found that dynamic light scattering experiments provide similar information on the gelling time, about 30 hours, to that obtained from rotational rheology and oscillatory rheology. This result confirms the validity of light scattering, which is a noninvasive technique, to characterize mechanical properties of time evolving hybrid materials through nonperturbative and well-controlled experiments.

\section{Introduction}

Stone coating has been extensively used lastly to conserve our artistic stonework. In this direction, an enormous effort has been done to design materials that, without changing the visual appearance of the stone, provide it with consolidant properties and protection against environmental hazards such as erosion, humidity, and microbial attack [1]. In this regard, organically modified silicates (ORMOSILs) seem to fulfill most of these requirements, except antimicrobial activity, and they have been successfully used for stone preservation $[2,3]$. As with all alkoxysilane-based products, these materials undergo a sol-gel transition. They are applied on the surface when the material is a liquid with low viscosity, and then the product polymerizes spontaneously inside the porous structure of the stone creating a polymer network of silica [4]. During polymerization two reactions take place: hydrolysis of alkoxy groups to generate silanol ones and condensation between silanol groups of the product and/or between these groups and those present in the silicate minerals. TEOS polymeric precursor is the most commonly used for stone consolidation, mixed with solvents to homogenize the water-alkoxysilane mixture, and with a catalyst to increase the rate of the hydrolysis reaction. In our case, the hybridization of the silica gel is done with polydimethylsiloxane (PDMS) since it increases flexibility and strength of the resulting coating $[5,6]$ and accelerates the gelation process $[7,8]$. Moreover, adding PDMS to the silicon alkoxide does not increase the viscosity of the initial sol, which makes its application over the stone and its penetration equally facile. However, most of the gels obtained from commercial formulations are highly brittle and crack easily. This inconvenience is circumvented by adding a nonionic surfactant (n-octylamine) to the synthesis as shown by the group led by Mosquera $[9,10]$. The presence of this nonionic surfactant accelerates the gelling process [8] and avoids gel fracture during the drying stage due to the formation of a more uniform size distribution of mesopores 
[11]. In this way, ORMOSIL products with hydrofugant and consolidant properties can be obtained by mixing TEOS and a poly(dimethylsiloxane) with terminal $\mathrm{OH}$ groups, PDMS, under the presence of a surfactant $[10,12]$. To provide the material with antimicrobial activity, here we propose the addition of $\mathrm{ZnO}$ nanoparticles $[13,14]$ to the aforementioned polymeric formulation [12]. We select these nanoparticles due to their confirmed chemical stability, biocompatibility, and bioactivity [15] and to their limited use in materials for stone conservation. Moreover, these nanoparticles may also provide superhydrophobicity to the material [16], which is also highly desired for coatings used for stone protection. Recently, it has been shown that a similar product (prehydrolyzed TEOS, water, and n-octylamine) acquires biocide action after adding to the synthesis $\mathrm{CuO}$ nanoparticles [17].

The study of the sol-gel process existing in this kind of products has an enormous importance, in particular, the sol-gel transition point, to characterize the formation of the network structure that is responsible for the potential applications of such materials. Furthermore, the gelling time is a crucial parameter to evaluate their suitability since gelation cannot be too fast, to facilitate its application over the stone and its penetrability, nor too slow, to avoid evaporation of volatile molecules. During the sol-gel process, there is an enhancement of the viscosity due to the cluster formation, and then, at the gel point, the emergence of a threedimensional network provokes a divergence of the viscosity and the appearance of elasticity in the material, process that is also known as "amorphous solidification" or "gelation" transition [18]. Indeed, after this point, it is said that a gel is formed, and an infinite cluster spans the sample that is capable of sustaining stress provoking a change of its macroscopic properties, where material elasticity overpasses viscosity. The determination of the gel point can be done employing several techniques. For example, Raman spectroscopy may provide useful information about the polymerization process underlying the gel formation, and it has been applied to several materials with coatings applications as polyalkyl siloxanes [19], biopolymers [20], among others. Alternatively, other techniques as rotational [21] or oscillatory $[22,23]$ rheological tests are required to follow the mechanical properties during gelation. However, rheology possesses some uncertainty in the determination of the gel point due to, on the one hand, the intrinsic difficulty of evaluating the divergence of one physical magnitude and, on the other hand, the shear applied to the material in this kind of measurements can perturb the sol-gel transition we want to characterize. An alternative technique to study the mechanical properties of a given medium is microrheology [24]. This technique is based on the fact that the motion of particles embedded in a given medium is related to its mechanical properties. In general, there are several techniques to obtain information on the dynamics of probe particles immersed in viscoelastic systems. One of them is based on dynamic light scattering [25]. Sometimes, the scatterers are introduced intentionally into the sample and, other times, the system possesses intrinsic inclusions, which can be used as probe particles. The advantage of microrheology is that the viscoelastic response of the medium is measured in the linear regime since the stress applied to the material by the probe particle is due to its Brownian motion.

The previously mentioned techniques can be used to characterize the sol-gel process under interest, but they not always agree with each other [26]. To discard possible differences among different techniques, we characterize the gel point of our novel material by rheological measurements and light scattering techniques. The advantage of using the latter technique is that it is noninvasive and, in our case, we use the $\mathrm{ZnO}$ particles present in the system as tracer particles with no need of introducing additional optical probes. Our product could be a novel alternative since the sol-gel route here involved produces homogenous crack-free and transparent nanomaterials that could combine multiple protection mechanisms for building stone (consolidant, hydrofugant, and biocidal effects).

\section{Materials and Methods}

2.1. Sol-Gel Preparation. In this work, we synthesize silica$\mathrm{ZnO}$ organic-inorganic nanocomposites by mixing $\mathrm{ZnO}$ nanoparticles with a silica oligomer in the presence of a nonionic surfactant (n-octylamine) and of a hydroxyl-terminated polydimethylsiloxane. These nanocomposites integrate $\mathrm{ZnO}$ nanoparticles in a mesoporous silica-based matrix prepared according to a synthesis route previously published [12]. The nanomaterial starts from a sol containing TES $40 \mathrm{WN}$ (from Wacker), which is a prehydrolyzed tetraethyl orthosilicate, in the presence of n-octylamine (from Aldrich). According to its technical data sheet, TES $40 \mathrm{WN}$ (hereafter TES40) is a mixture of monomeric and oligomeric ethoxysilanes, whose average chain length is approximately $5 \mathrm{Si}-\mathrm{O}$ units. The $\mathrm{ZnO}$ nanopowders (from Nanostructured \& Amorphous Materials, Inc.) have an average primary particle size of 20 $\mathrm{nm}$ provided by the supplier. PDMS (from Gelest) has a polymerization degree of 12 (molar mass 400-700) and an $\mathrm{OH}$ percentage ranging from 4.5 to $7.5 \% \mathrm{w} / \mathrm{w}$. The synthesis of our organic-inorganic nanocomposite is as follows. Firstly, we prepare by vigorous stirring an aqueous solution of $n-$ octylamine at a concentration of $1.57 \mathrm{~mol} \cdot \mathrm{dm}^{-3}$, which is significantly higher than its critical micellar concentration. Then the aqueous solution of n-octylamine is mixed with TES40 under high-power ultrasonic agitation $\left(60 \mathrm{~W} \cdot \mathrm{cm}^{-3}\right)$ for 10 minutes. The resulting molar ratio of TES40 to $\mathrm{n}$ octylamine and water are 1: $5.3 \times 10^{-4}$ and 1: $133.6 \times 10^{-4}$, respectively. Next, under this ultrasonic agitation, $\mathrm{ZnO}$ particles are incorporated up to a weight fraction of $0.005 \% \mathrm{w} / \mathrm{w}$. Finally, keeping the ultrasonic agitation, PDMS is added to the starting sol drop by drop up to a concentration of $10.4 \%$ $\mathrm{v} / \mathrm{v}$.

2.2. Rheology Measurements. The viscosity and viscoelastic moduli of our suspensions are measured with an MCR 502 rheometer (Anton Paar) by using a cone-plate geometry of 50 $\mathrm{mm}$ of diameter and a cone angle of one degree. The steady flow curves to determine the shear viscosity are obtained by rotational tests with controlled shear rate. The time needed for the sample to reach constant conditions for each shear rate, and consequently to perform the measurement, is 
determined automatically by the rheometer after recording constant consecutive values of the viscosity. The frequency dependence of the viscoelastic moduli is measured in the oscillatory mode in the linear viscoelastic regime by applying a strain of $0.3 \%$. This strain assures a linear response of the sample for all measurements, confirmed by the dependence of the storage and loss moduli with deformation measured at a frequency of $10 \mathrm{rad} / \mathrm{s}$.

2.3. Light Scattering Technique. The size of the $\mathrm{ZnO}$ colloidal particles is measured with a Zetasizer ZS90 (Malvern Inst.). This device estimates the size of particles immersed in a fluid from the autocorrelation function of the scattered intensity by means of the well-known technique dynamic light scattering (DLS). The fluctuation of the intensity scattered by the particles is related to their motion and the intensity correlation function minus one, $g_{I}(\tau)-1$, in case of colloidal systems composed of particles having the same size and immersed in purely viscous fluids, decays exponentially with time [27]:

$$
g_{I}(\tau)-1=A e^{-\tau / \tau_{c}}
$$

where $\tau_{\mathrm{C}}$ is the characteristic decay time of the exponential function and is equal to

$$
\tau_{C}=\frac{1}{2 D_{0} q^{2}}
$$

and here $q$ is the scattering vector given by

$$
q=\frac{4 \pi n}{\lambda} \sin \left(\frac{\theta}{2}\right)
$$

The scattering vector depends on the refractive index, $n$, of the media where the particles are inmersed, the wavelength of the laser, $\lambda=632.8 \mathrm{~nm}$, and the scattering angle, $\theta=90^{\circ}$. $\mathrm{D}_{0}$ is the free particle diffusion coefficient given by the StokesEinstein relation that, in case of a sphere, takes the form

$$
D_{0}=\frac{\kappa_{B} T}{6 \pi \eta a}
$$

where $T$ is the temperature of the suspending medium, $\eta$ is its viscosity, and $a$ is the particle radius. Note that this expression can be equally used to determine the size of probe particles, when the viscosity of the fluid is known, or to determine the viscosity of a certain fluid by using particles of known diameter. The latter application is the most straightforward case of the technique called microrheology.

\section{Results and Discussion}

First, we measure the size of $\mathrm{ZnO}$ nanoparticles at $25^{\circ} \mathrm{C}$ when they are immersed in TES40 and in the system of interest, that is, after adding PDMS and the n-octylamine solution to the TES40, immediately after preparation. In both cases, the estimated sizes and polydispersity index, IPD, are equal to 360 $\pm 30 \mathrm{~nm}$ and $0.20 \pm 0.05$, respectively. From this result, we prove that the particles are forming nanoclusters of similar size that are uniformly distributed in the precursor media,
TABLE 1: Viscosities measured at $\mathrm{T}=25^{\circ} \mathrm{C}$ by rotational rheology of the main component of the sample, TES40, and after adding the different components, TES40+PDMS, TES40+PDMS+n-octylamine solution and TES40+PDMS+n-octylamine solution $+\mathrm{ZnO}$ particles.

\begin{tabular}{lc}
\hline Sample & $\eta[\mathrm{mPa} \cdot \mathrm{s}]$ \\
\hline TES40 & $4.28 \pm 0.13$ \\
TES40+PDMS & $4.44 \pm 0.07$ \\
TES40+PDMS+n-octylamine solution & $4.56 \pm 0.14$ \\
TES40+PDMS+n-octylamine solution+ZnO & $4.6 \pm 0.4$ \\
\hline
\end{tabular}

TES40, and that the addition of the rest of components to the product does not affect such homogeneity.

The size of the $\mathrm{ZnO}$ particles is determined by using the Stokes-Einstein relation, where the viscosity is needed. The viscosities of the different suspending media are measured by rotational rheology. We measure the viscosity of the main component of the media, TES40, and then we characterize how it changes as the rest of the reagents, PDMS, solution of n-octylamine, and colloidal particles are added. The mean viscosities measured at $25^{\circ} \mathrm{C}$ are shown in Table 1 , where the error is estimated as the sample standard deviation. As we can see, the addition of the different components to the precursor media slightly augments its viscosity, keeping the material penetrability into the stone.

After the sample preparation, once the system is ultrasonicated, its mechanical properties are followed in time at a temperature of $25^{\circ} \mathrm{C}$. By performing rotational tests, we observe that the viscosity does not depend on shear rate during the initial hours after preparation in the interval of shear rates analyzed, showing the typical behavior of Newtonian fluids. However, after around 28 hours, viscosity starts to show a slight dependence on shear rate due to the polymerization process. During these first 28 hours, the viscosity only augments slightly but, after this waiting time, the viscosity increases very abruptly as shown in Figure 1(b), where we plot the dependence on reaction time of the viscosity normalized by its initial value measured at zero time, just after sample preparation. This sudden increase of the viscosity on time is a hallmark of the sol-gel transition, caused by the formation of an interconnected network that spans the sample. In fact, it is by performing a power law fit to the data (continuous line in Figure 1(b)) by using the model

$$
\frac{\eta(t)}{\eta_{0}}=\left(1-\frac{t}{t_{g e l}}\right)^{-s} \text {. }
$$

We get that the gelation time $t_{\text {gel }}=(30.2 \pm 0.1)$ hours and $s$ $=(0.72 \pm 0.02)$. This expression has been previously used to describe very well the time dependence of the viscosity in sol-gel transitions [28]. The value obtained for the critical exponent is quite close to the experimental value 0.75 found in silica-siloxane mixtures [29] and to the expected value of 0.7 predicted by the percolation theory [28].

Up to now, our characterization is based on rotational rheology where shear is applied to the sample and, consequently, this perturbation may alter the gelification process. For this reason, we also characterize the sol-gel transition 


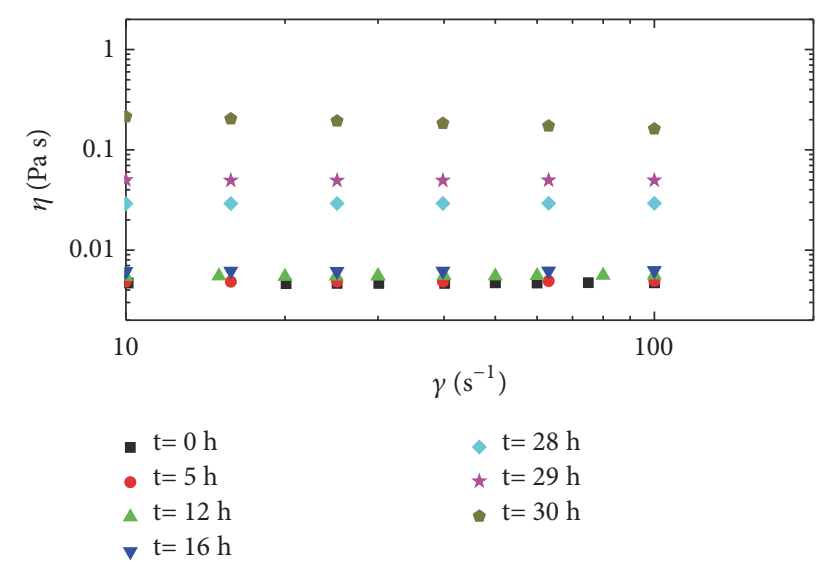

(a)

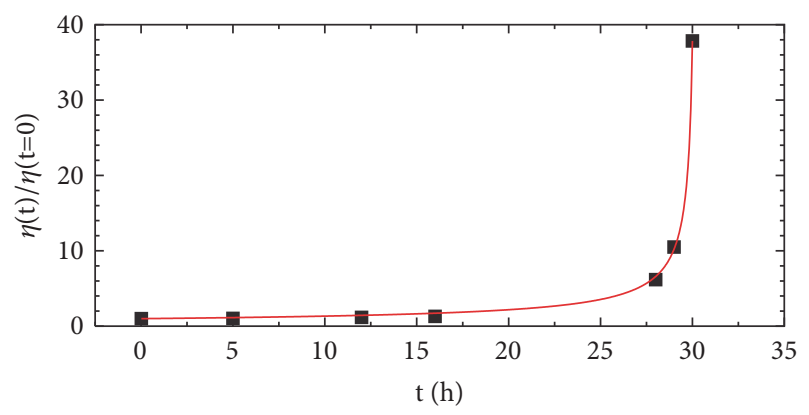

(b)

FIgURE 1: (a) Viscosity as a function of shear rate measured at different times after sample preparation at $\mathrm{T}=25^{\circ} \mathrm{C}$ : black squares $(\mathrm{t}$ $=0 \mathrm{~h})$, red circles $(t=5 \mathrm{~h})$, green up triangles $(t=12 \mathrm{~h})$, blue down triangles $(t=16 \mathrm{~h})$, cyan diamonds $(t=28 \mathrm{~h})$, pink stars $(t=29 \mathrm{~h})$, and dark yellow pentagon $(t=30 \mathrm{~h})$. (b) Normalized viscosity measured as a function of time (squares) and power law fit, $\left(1-\left(t / t_{\text {gel }}\right)\right)^{-s}$, to the experimental data, where $t_{\text {gel }}$ and $s$ are fitting parameters (continuous line).

by oscillatory rheology where we determine the frequency dependence of the viscoelastic moduli on the reaction time by applying an oscillatory stress whose amplitude is very small to assure the linear viscoelastic regime. The measured viscoelastic moduli are plotted as a function of frequency for different reaction times in Figure 2(a). For all the times investigated both moduli increase with frequency. At the initial times, the viscous modulus (hollow symbols) is higher than the elastic one (solid symbols) for all the frequencies under investigation up to a certain time, where the elastic component overcomes the viscous one, which shows the existence of a gel. This inversion point is placed between 31 and 32 hours and, precisely in this interval, we can observe in Figure 2(b) the power law dependence of the elastic and viscous moduli with frequency $G^{\prime} \sim G^{\prime \prime} \sim \omega^{n}$, where $n$ is known as the critical viscoelastic exponent. This power law frequency dependence of the dynamic moduli resembles the behavior of a weak gel and serves to identify the formation of an incipient gel at the gel point $[22,23]$, indicating the presence of a three-dimensional network and, consequently, the transition from a liquid to a solid-like behavior.

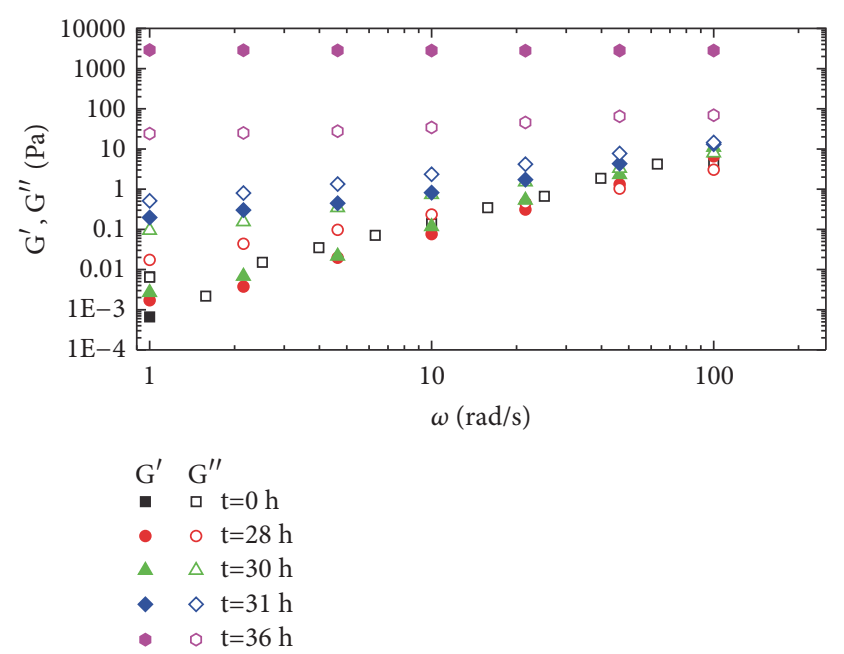

(a)

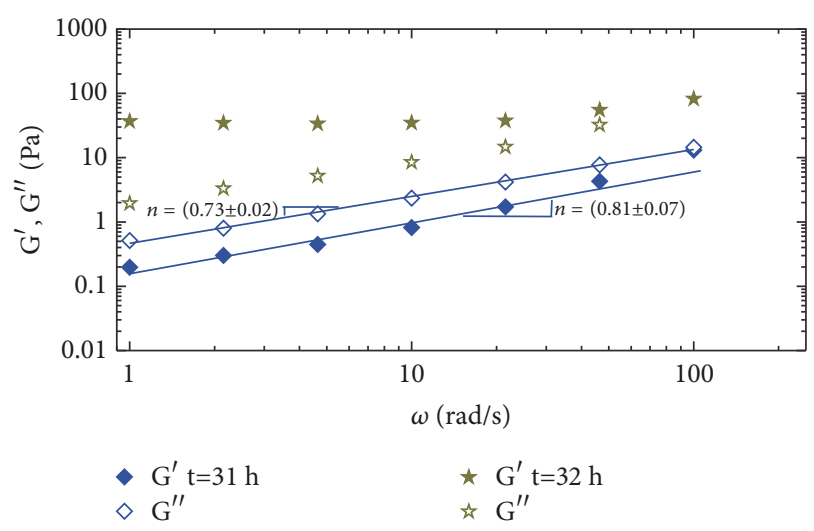

(b)

FIGURE 2: (a) Elastic (solid symbols) and viscous (hollow symbols) moduli measured as a function of frequency at different reaction times at $\mathrm{T}=25^{\circ} \mathrm{C}$ : immediately after sample preparation (black squares), after 28 hours (red circles), after 30 hours (green triangles), and after 36 hours (pink hexagons). (b) Elastic and viscous moduli measured 31 hours after sample preparation (blue diamonds) along their corresponding fits to a power law $\mathrm{G} \sim \omega^{\mathrm{n}}$ (continuous lines) and 32 hours after sample preparation (dark yellow stars).

In our case, the critical viscoelastic exponents $n=$ $(0.81 \pm 0.07)$ obtained from the frequency dependence of the elastic modulus, i.e., $\mathrm{G}^{\prime}(\omega) \sim \omega^{0.81 \pm 0.07}$ and $n=(0.73 \pm 0.02)$, from the frequency analysis of the viscous modulus, G” $(\omega) \sim$ $\omega^{0.73 \pm 0.02}$, are in agreement with the interval of values found in the literature. For branched polymer, as in our case, it is expected that this value is in the interval that goes from 0.66 to 1 [30] and it is close to the value 0.67 derived from percolation theory [31]. The values so obtained for the critical exponents, considering their uncertainty, are consistent between them, which guarantees that the gel point is quite close to this time. In fact, the next measurement, performed after 32 hours, shows a solid-like behavior since for all frequencies investigated the elastic modulus is higher than the viscous one, and at low frequencies the elastic modulus tends to a finite value, indicative of the gel nature of the sample. 


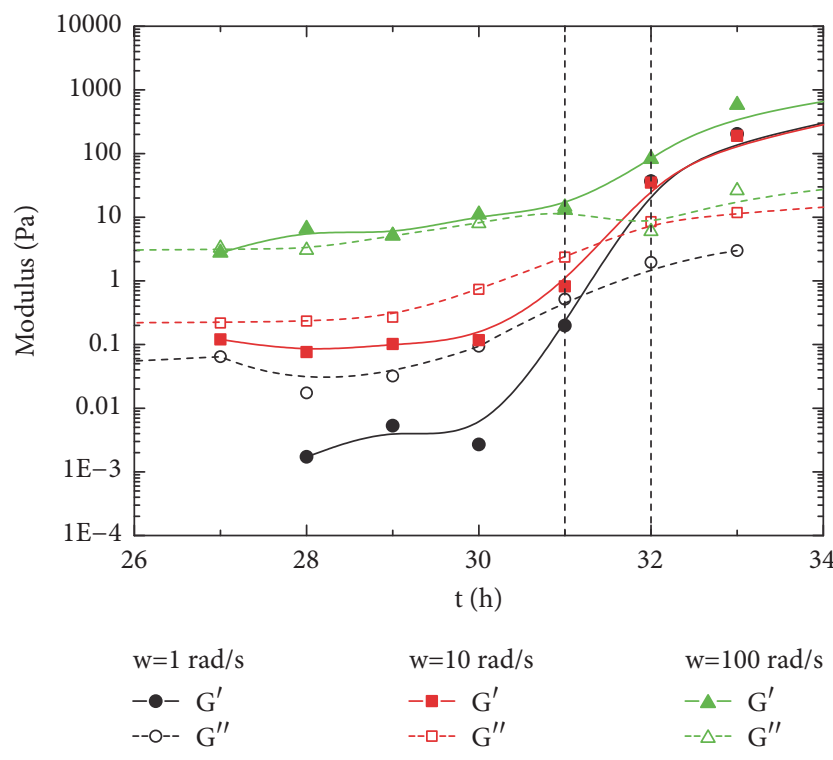

FIgURE 3: Elastic (solid symbols) and viscous (hollow symbols) moduli measured at frequencies of $1 \mathrm{rad} / \mathrm{s}$ (black circles), $10 \mathrm{rad} / \mathrm{s}$ (red squares), and $100 \mathrm{rad} / \mathrm{s}$ (green triangles) as a function of time. Lines are guides to the eye. Dashed vertical lines indicate the time interval where the crossover of G'-G" occurs.

As the gel evolves, elasticity increases faster than viscosity at all frequencies and, after 36 hours, the system resembles the mechanical response of a fully developed network where the elastic modulus hardly depends on frequency and is two orders of magnitude larger than the viscous one, which are typical features of strong gels. From this finding, we can guarantee that the sol to gel transition occurs between 31 and 32 hours after sample preparation. This gel time also coincides with the time for which the elastic modulus (solid symbols) equals the viscous one (hollow symbols) in a broad interval of frequencies (see Figure 3), criterion also established by Tung [21] to determine the gel point. In this figure, the time dependence of the elastic and viscous moduli is plotted for 3 different frequencies: $1 \mathrm{rad} / \mathrm{s}$ (black circles), $10 \mathrm{rad} / \mathrm{s}$ (red squares), and $100 \mathrm{rad} / \mathrm{s}$ (green triangles), and we can see here that the time when $G^{\prime}=G^{\prime \prime}$ is placed between 31 and 32 hours (vertical lines in Figure 3). As we can see the values of the gel time obtained by both methods coincide, showing the validity of our analysis. Moreover, the critical viscoelastic exponents found in our system is in accordance with the interval $0.6-0.8$ reported by other authors for this critical exponent in tetraethyl orthosilicate based hybrid solutions $[32,33]$.

To deepen the study of the gel point, we proceed with the technique previously described, known as microrheology. Passive microrheology is a technique that allows determination of the viscoelastic properties of materials with no need of applying external forces to the sample, since the only forces exerted on the system are those due to the thermal motion of the tracer particles immersed in the medium. In this way, the deformation energy in the system is of the order of the thermal energy that is responsible for the Brownian motion of the probe particles, guaranteeing the linear viscoelastic regime. Sometimes, the inconvenience of this technique is either the difficulty of finding suitable tracers since they must not alter the physical properties of the medium that will be characterized or the mechanical agitation occasionally needed to disperse the particles. In our case, we can circumvent these problems since there is no need to introduce the tracer particles to the system, by using as tracers the $\mathrm{ZnO}$ particles that are already present in our material. In microrheology experiments based on light scattering, the autocorrelation function of the intensity scattered by the tracers is measured. Here it is critical to ensure that most of the light scattered by the sample comes from the optical probes, as it happens in our system due to the $\mathrm{ZnO}$ particle concentration used in the sample preparation, and the low scattering power of the suspending medium. In this case, the autocorrelation function only contains information on the tracer motion. For example, when the probes particles are immersed in a Newtonian fluid, the autocorrelation function of the intensity minus one decays exponentially and its decay time is proportional to the medium viscosity; see (2) and (4). In our case, we use this relation to follow the time evolution of the viscosity of our polymeric material by measuring the autocorrelation function of the intensity scattered by the $\mathrm{ZnO}$ particles immersed in the dispersion of interest, as a function of reaction time, and data are shown in Figure 4(a). Here we can see that for the initial 30 hours the intensity autocorrelation function decays exponentially, meaning that the suspending medium is mostly viscous, and the only difference that can be appreciated is the increase of the decay time as the system evolves. This increase of the decay time, since the size particle is kept constant along the experiment, could only be due to an increase of the medium viscosity. However, for waiting times longer than 31 hours, we can appreciate that the autocorrelation function does not decay to zero. This feature is due to the fact that the $\mathrm{ZnO}$ tracer particles immersed in the polymer dispersion are dynamically arrested; thus they cannot move distances that allow light decorrelation. The dynamic arrest of the tracers, inferred from these measurements, is a clear evidence of the emergence of a three-dimensional network in the suspending medium. Quantitatively, this liquid to solid transition can be analyzed by plotting the decay time of $g_{I}(\tau)-1$ normalized by the decay time of the autocorrelation function measured just after sample preparation as a function of waiting time for the ergodic samples, as plotted in Figure 4(b). The ratio between these two decay times corresponds precisely to the system viscosity normalized by the viscosity of the sample at time zero. Here we can notice that the time behavior of the sample viscosity obtained by light scattering resembles that measured by rotational rheology, which was plotted in Figure 1(b). In fact, the experimental time dependence of the viscosity shown in the figure can be very well described by the same power law, (5). From the fit, we obtain that the gelation time is $\mathrm{t}_{\mathrm{gel}}=(30.1 \pm 0.2)$ hours, and $\mathrm{s}=(0.62 \pm 0.03)$, values that are very similar to those found from rotational rheometry.

This gel time value is smaller than the one obtained for the polymeric matrix without nanoparticles, which is around 35 hours. Therefore, as found for $\mathrm{CuO}$ nanoparticles in this type 


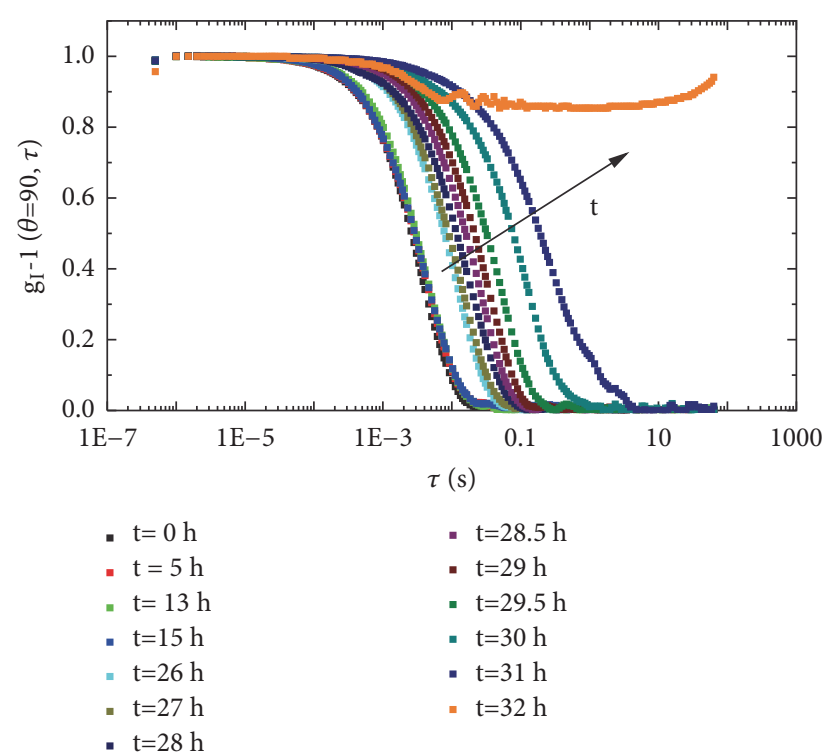

(a)

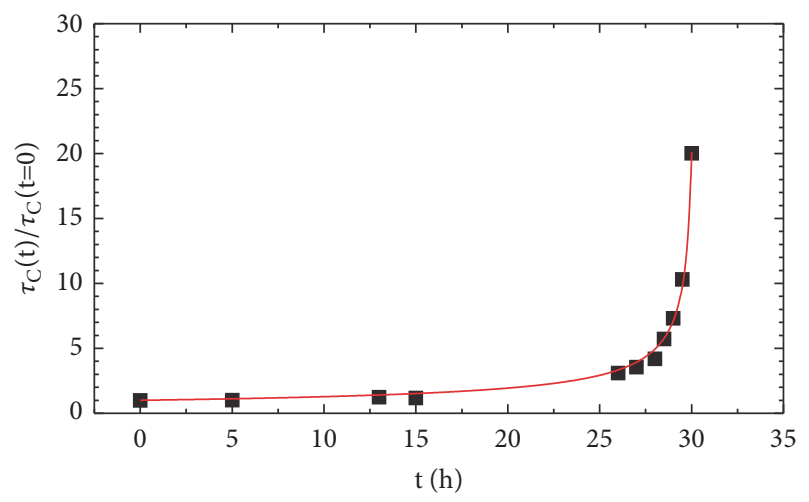

(b)

FIGURE 4: (a) Intensity autocorrelation function minus one measured as a function of time. (b) Ratio of the characteristic times obtained from the analysis of the autocorrelation functions shown in (a) normalized by the initial decay time measured at $t=0 \mathrm{~h}$. Line is a fit to a critical power law: $\tau_{C}(t) / \tau_{0}=\left(1-\left(t / t_{\text {gel }}\right)\right)^{-s}$, where $\tau_{0}$ is the decay time of the autocorrelation at time $t=0 \mathrm{~h}$ and $\mathrm{t}_{\text {gel }}$ and $\mathrm{s}$ are fitting parameters.

of formulations [17], we can think this acceleration is due to the favoured release of $\mathrm{Zn}^{2+}$ ions at the basic conditions of the initial sol, which accelerates the hydrolysis of the ethoxy groups, and to the fact that $\mathrm{Zn}^{2+}$ may also interact with [SiO $\left.(\mathrm{OH})_{\mathrm{X}}\right]^{-}$intermediate species, which promotes the formation of $\mathrm{SiO}_{2}$ nuclei by condensation.

\section{Conclusions}

In this work, we present a study on the gelation process of an organically modified silicate that consists of a silica polymeric precursor mixed with n-octylamine and an organosilicon compound, PDMS, which has application in restoration and conservation of building substrates. During material preparation, $\mathrm{ZnO}$ particles are introduced into the system in order to obtain stone treatments that, besides their consolidant and hydrofugant properties, present biocide activity $[12,13]$. Moreover, the resulting product shows low viscosity that ensures an easy and homogenous application over the stone and a reasonable reaction time to reach the gel point. Despite the importance of the gel point in material science, its determination is still controversial since the sol-gel transition can be found at different points depending on the method employed. For this reason, we have determined the gel point of our sol-gel process by different techniques: rotational and oscillatory rheology and light scattering experiments. The gel point estimated from the divergence of the viscosity, measured by rotational rheology, is $t_{\text {gel }}=(30.2 \pm 0.1)$ hours, and it is very similar to that obtained from the time where the frequency dependence of the viscoelastic moduli obeys a power law $t_{\text {gel }}=31$ hours and from the crossing point where the elastic modulus equals the viscous one, between 31 and 32 hours. The critical viscoelastic exponent found from the frequency dependence of the storage and loss moduli at the gel point is around $(0.81 \pm 0.07)$ and $(0.73 \pm 0.02)$, respectively, values similar to the expected value of 0.67 obtained from percolation theory. Furthermore, by performing light scattering experiments where the sample is not perturbed, we obtain $t_{\text {gel }}=(30.1 \pm 0.2)$ hours that agrees with those found from rheological measurement. Here we show how light scattering, a noninvasive technique, can be applied to study a new family of gelling products that contain nanoparticles for a given application by using them as tracers to characterize the mechanical properties of such products. Although biocide activity of the composite presented here has not yet been proved, this work is an attempt to obtain stone coatings with biocide activity that do not alter the stone appearance and still have consolidating and hydrofugant properties. In this direction, further studies using different $\mathrm{ZnO}$ particle concentrations and different size of particles are currently under development.

\section{Data Availability}

The data used to support the findings of this study are available from the corresponding author upon request.

\section{Conflicts of Interest}

The authors declare that there are no conflicts of interest regarding the publication of this paper.

\section{Acknowledgments}

The authors thank Conacyt for the financial support: C. HaroPérez (project 166645), Juan F. Illescas, and J. Aguilar-Pliego (Project 257449) and PRODEP.

\section{References}

[1] M. Hosseini and I. Karapanagiotis, Advanced Materials for the Conservation of Stone, Springer International Publishing, Cham, 2018. 
[2] R. Zárraga, J. Cervantes, C. Salazar-Hernandez, and G. Wheeler, "Effect of the addition of hydroxyl-terminated polydimethylsiloxane to TEOS-based stone consolidants," Journal of Cultural Heritage, vol. 11, no. 2, pp. 138-144, 2010.

[3] I. Karapanagiotis, A. Pavlou, P. N. Manoudis, and K. E. Aifantis, "Water repellent ORMOSIL films for the protection of stone and other materials," Materials Letters, vol. 131, pp. 276-279, 2014.

[4] G. Wheeler, Alkoxysilanes and the consolidation of stone, The Getty Conservation Institute,.

[5] H.-H. Huang, B. Orler, and G. L. Wilkes, "Structure-Property Behavior of New Hybrid Materials Incorporating Oligomeric Species into Sol-Gel Glasses. 3. Effect of Acid Content, Tetraethoxysilane Content, and Molecular Weight of Poly(dimethylsiloxane)," Macromolecules, vol. 20, no. 6, pp. 1322-1330, 1987.

[6] S. J. Kramer, F. Rubio-Alonso, and J. D. Mackenzie, "Organically Modified Silicate Aerogels, "Aeromosils", Materials Research Society - Proceedings, vol. 435, 1996.

[7] J. Wen and J. E. Mark, "Sol-Gel preparation of composites of poly(dimethylsiloxane) with $\mathrm{SiO} 2$ and $\mathrm{SiO} 2 / \mathrm{TiO} 2$, and their mechanical properties," Polymer Journal, vol. 27, no. 5, pp. 492$502,1995$.

[8] J. F. Illescas and M. J. Mosquera, "Surfactant-synthesized PDMS/silica nanomaterials improve robustness and stain resistance of carbonate stone," The Journal of Physical Chemistry C, vol. 115, no. 30, pp. 14624-14634, 2011.

[9] M. J. Mosquera, D. M. De Los Santos, A. Montes, and L. ValdezCastro, "New nanomaterials for consolidating stone," Langmuir, vol. 24, no. 6, pp. 2772-2778, 2008.

[10] M. J. Mosquera, D. M. De Los Santos, and T. Rivas, "Surfactantsynthesized ormosils with application to stone restoration," Langmuir, vol. 26, no. 9, pp. 6737-6745, 2010.

[11] D. S. Facio, M. Luna, and M. J. Mosquera, "Facile preparation of mesoporous silica monoliths by an inverse micelle mechanism," Microporous and Mesoporous Materials, vol. 247, pp. 166-176, 2017.

[12] J. F. Illescas and M. J. Mosquera, "Producing surfactant-synthesized nanomaterials in situ on a building substrate, without volatile organic compounds," ACS Applied Materials \& Interfaces, vol. 4, no. 8, pp. 4259-4269, 2012.

[13] S. A. Ruffolo, M. F. La Russa, M. Malagodi, C. Oliviero Rossi, A. M. Palermo, and G. M. Crisci, "ZnO and $\mathrm{ZnTiO} 3$ nanopowders for antimicrobial stone coating," Applied Physics A: Materials Science \& Processing, vol. 100, no. 3, pp. 829-834, 2010.

[14] O. M. El-Feky, E. A. Hassan, S. M. Fadel, and M. L. Hassan, "Use of $\mathrm{ZnO}$ nanoparticles for protecting oil paintings on paper support against dirt, fungal attack, and UV aging," Journal of Cultural Heritage, vol. 15, no. 2, pp. 165-172, 2014.

[15] N. Ditaranto, I. D. Van Der Werf, R. A. Picca et al., "Characterization and behaviour of $\mathrm{ZnO}$-based nanocomposites designed for the control of biodeterioration of patrimonial stoneworks," New Journal of Chemistry, vol. 39, no. 9, pp. 6836-6843, 2015.

[16] P. N. Manoudis and I. Karapanagiotis, "Modification of the wettability of polymer surfaces using nanoparticles," Progress in Organic Coatings, vol. 77, no. 2, pp. 331-338, 2014.

[17] R. Zarzuela, M. Carbú, M. L. A. Gil, J. M. Cantoral, and M. J. Mosquera, "CuO/SiO2 nanocomposites: A multifunctional coating for application on building stone," Materials and Corrosion, vol. 114, pp. 364-372, 2017.

[18] J. Douglas, "Weak and Strong Gels and the Emergence of the Amorphous Solid State," Gels, vol. 4, no. 1, p. 19, 2018.
[19] D. Lampakis, P. N. Manoudis, and I. Karapanagiotis, "Monitoring the polymerization process of Si-based superhydrophobic coatings using Raman spectroscopy," Progress in Organic Coatings, vol. 76, no. 2-3, pp. 488-494, 2013.

[20] T. Stößer, C. Li, J. Unruangsri et al., "Bio-derived polymers for coating applications: Comparing poly(limonene carbonate) and poly(cyclohexadiene carbonate)," Polymer Chemistry, vol. 8, no. 39, pp. 6099-6105, 2017.

[21] C. M. Tung and P. J. Dynes, "Relationship between viscoelastic properties and gelation in thermosetting systems," Journal of Applied Polymer Science, vol. 27, no. 2, pp. 569-574, 1982.

[22] H. H. Winter and F. Chambon, "Analysis of Linear Viscoelasticity of a Crosslinking Polymer at the Gel Point," Journal of Rheology, vol. 30, no. 2, pp. 367-382, 1986.

[23] H. H. Winter, "Can the gel point of a cross-linking polymer be detected by theG? -G? crossover?" Polymer Engineering \& Science, vol. 27, no. 22, pp. 1698-1702, 1987.

[24] T. G. Mason and D. A. Weitz, "Optical measurements of frequency-dependent linear viscoelastic moduli of complex fluids," Physical Review Letters, vol. 74, no. 7, pp. 1250-1253, 1995.

[25] F. Scheffold, S. Romer, F. Cardinaux et al., "New trends in optical microrheology of complex fluids and gels," Progress in Colloid \& Polymer Science, vol. 141, 2004.

[26] S. Richter, V. Boyko, R. Matzker, and K. Schröter, "Gelation studies: Comparison of the critical exponents obtained by dynamic light scattering and rheology, 2a. A thermoreversible gelling system: Mixtures of xanthan gum and locust-bean gum," Macromolecular Rapid Communications, vol. 25, no. 16, pp. 1504-1509, 2004.

[27] R. Pecora, R. J. Nossal, R. Pecora, and A. V. Priezzhev, "Dynamic light scattering from macromolecules," in Proceedings of the OE/LASE'93: Optics, Electro-Optics, \& Laser Applications in Scienced Engineering, p. 2, Los Angeles, CA.

[28] P. Innocenzi, "Measuring the Sol-to-Gel Transition," in The Sol to Gel Transition, SpringerBriefs in Materials, pp. 51-61, Springer International Publishing, Cham, 2016.

[29] J.-P. Cohen-Addad, "Sol or gel-like behaviour of ideal silicasiloxane mixtures: percolation approach," Polymer Journal, vol. 33, no. 13, pp. 2762-2767, 1992.

[30] J. E. Martin, D. Adolf, and J. P. Wilcoxon, "Viscoelasticity near the sol-gel transition," Physical Review A: Atomic, Molecular and Optical Physics, vol. 39, no. 3, pp. 1325-1332, 1989.

[31] D. Stauffer, Introduction to Percolation Theory, Taylor \& Francis, Abingdon, UK, 1992.

[32] M. Jokinen, E. Györvary, and J. B. Rosenholm, "Viscoelastic characterization of three different sol-gel derived silica gels," Colloids and Surfaces A: Physicochemical and Engineering Aspects, vol. 141, no. 2, pp. 205-216, 1998.

[33] S.-Y. Kim, D.-G. Choi, and S.-M. Yang, "Rheological Analysis of the Gelation Behavior of Tetraethylorthosilane/Vinyltriethoxysilane Hybrid Solutions," Korean Journal of Chemical Engineering, vol. 19, no. 1, pp. 190-196, 2002. 

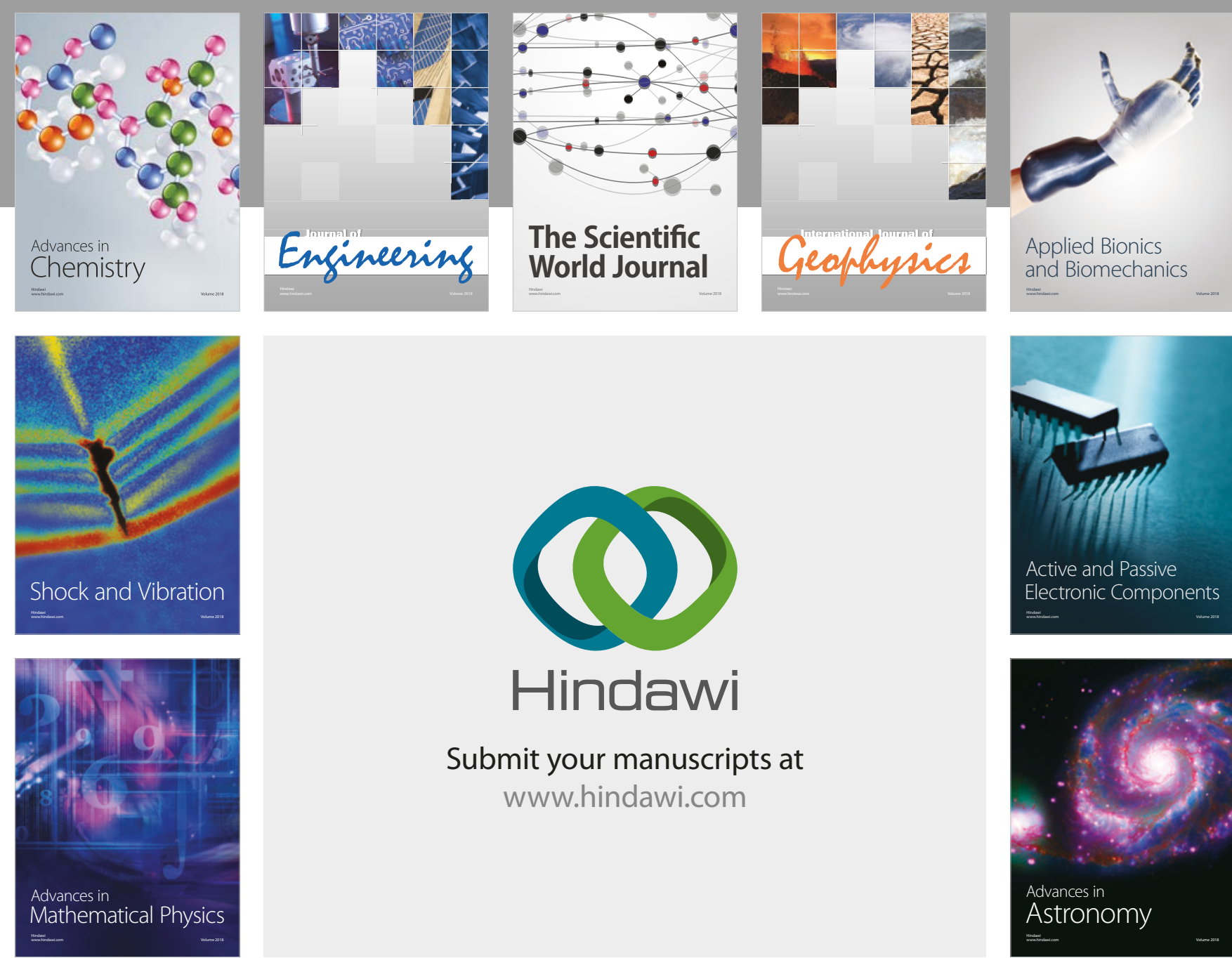

Submit your manuscripts at

www.hindawi.com

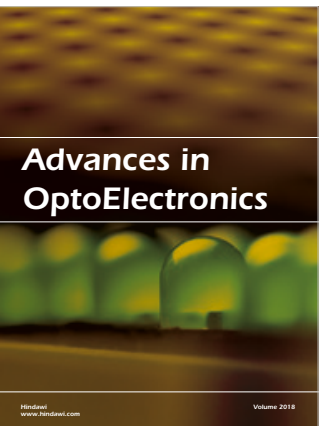

\section{Rotcting Machinery}
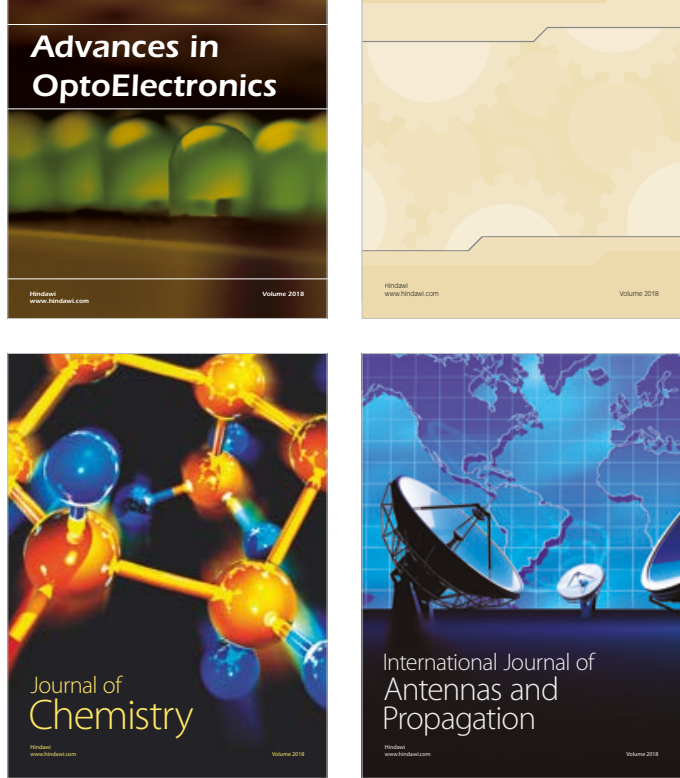

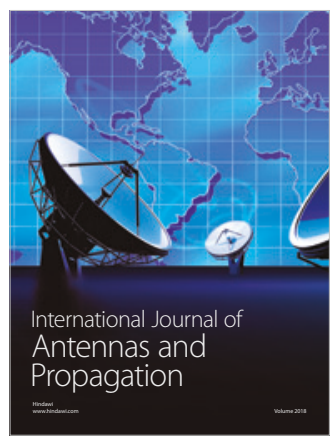

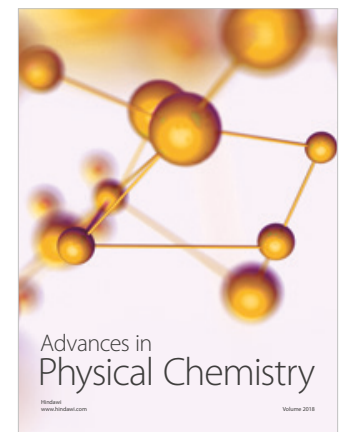

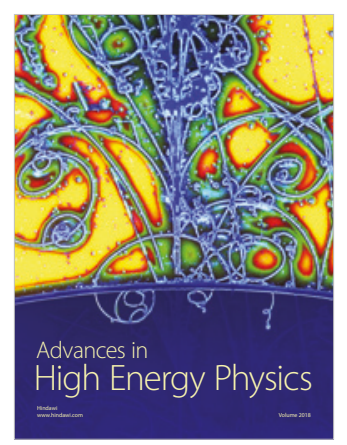

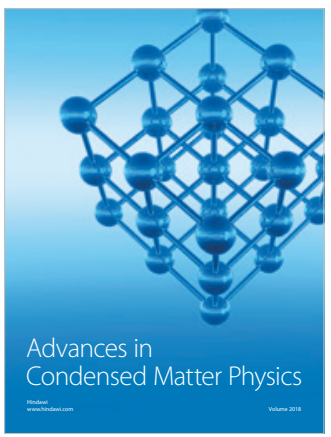

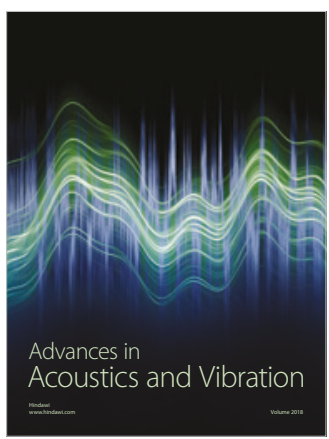

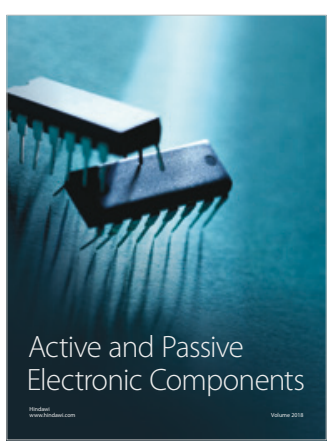
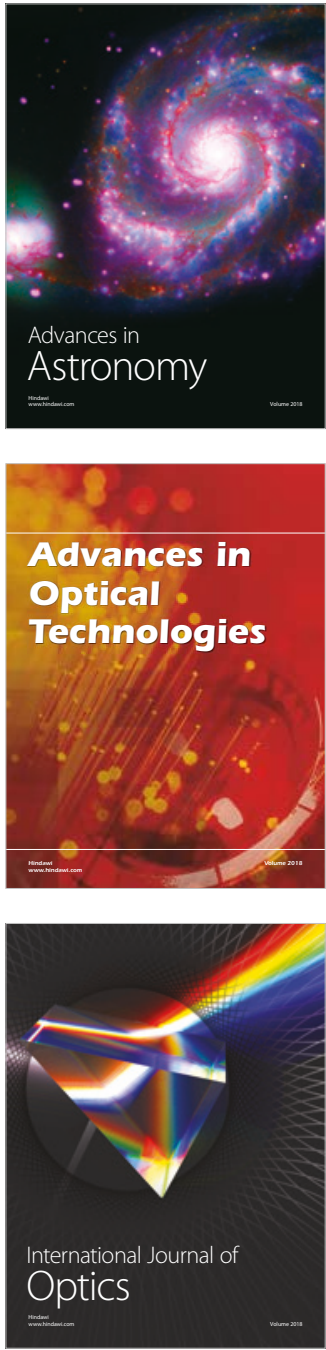